BMJ Surgery, Interventions, \& Health Technologies

\section{Perceptions of partial gland ablation for prostate cancer among men on active surveillance: a qualitative study}

To cite: Hur S, Tzeng M, CriccoLizza E, et al. Perceptions of partial gland ablation for prostate cancer among men on active surveillance: a qualitative study. BMJ Surg Interv Health Technologies 2021;3:e000068. doi:10.1136/ bmjsit-2020-000068

SH and MT are joint first authors.

Received 16 September 2020 Revised 18 February 2021 Accepted 05 April 2021

Check for updates

(c) Author(s) (or their employer(s)) 2021. Re-use permitted under CC BY-NC. No commercial re-use. See rights and permissions. Published by BMJ.

${ }^{1}$ Department of Urology, NewYork-Presbyterian/Weill Cornell Medicine, New York, New York, USA

${ }^{2}$ Department of Healthcare Policy and Research, Weill Cornell Medicine, New York, New York, USA

${ }^{3}$ Department of Pediatrics, Weill Cornell Medicine, New York,

New York, USA

${ }^{4}$ Department of Urology, David Geffen School of Medicine, Los Angeles, California, USA

${ }^{5}$ Department of Urology, Northwestern University Feinberg School of Medicine, Chicago, Illinois, USA

Correspondence to

Dr Jim Hu;

Jch9011@med.cornell.edu

\section{ABSTRACT}

Objectives Partial gland ablation (PGA) therapy is an emerging treatment modality that targets specific areas of biopsy-proven prostate cancer ( $\mathrm{PCa}$ ) to minimize treatment-related morbidity by sparing benign prostate. This qualitative study aims to explore and characterize perceptions and attitudes toward PGA in men with verylow-risk, low-risk, and favorable intermediate-risk PCa on active surveillance (AS).

Design 92 men diagnosed with very-low-risk, low-risk, and favorable intermediate-risk PCa on AS were invited to participate in semistructured telephone interviews on PGA. Setting Single tertiary care center located in New York City.

Participants 20 men with very-low-risk, low-risk, and favorable intermediate-risk PCa on AS participated in the interviews.

Main outcome measures Emerging themes on perceptions and attitudes toward PGA were developed from transcripts inductively coded and analyzed under standardized methodology.

Results Four themes were derived from 20 interviews that represent the primary considerations in treatment decision-making: (1) the feeling of psychological safety associated with low-risk disease; (2) preference for minimally invasive treatments; (3) the central role of the physician; (4) and the pursuit of treatment options that align with disease severity. Eleven men (55\%) expressed interest in pursuing PGA only if their cancer were to progress, while nine men (45\%) expressed interest at the current moment.

Conclusions Although an emerging treatment modality, patients were broadly accepting of PGA for $\mathrm{PCa}$, with men primarily debating the risks versus benefits of proactively treating low-risk disease. Additional research on men's preferences and attitudes toward PGA will further guide counseling and shared decision-making for PGA.

\section{INTRODUCTION}

Partial gland ablation (PGA) is an emerging modality in the treatment of prostate cancer (PCa) that allows cancer elimination while minimizing treatment-related morbidity.
The European Association of Urology (EAU) recognizes PGA as an investigational modality, and most studies focus on its role in men with very-low-risk to intermediaterisk PCa. ${ }^{12}$ Despite its potential, consensus on patient selection for PGA is lacking, as is intermediate to long-term outcomes on cancer control. $^{2-4}$

The Idea, Development, Exploration, Assessment and Long-term study classifies current evidence for PGA modalities as stage 2B in surgical innovation, showing need for comparative outcome evidence. ${ }^{5-7}$ PGA for PCa has been shown to have low rates of complications compared with the traditional whole gland treatments such as surgery or radiation therapy. ${ }^{2-4}$ However, post-PGA prostate-specific antigen (PSA) surveillance is unreliable due to background PSA produced by the untreated prostate. Therefore, monitoring with biopsies is the recommended post-PGA surveillance approach. ${ }^{8}$ Moreover, high-level evidence for PGA is lacking, and men face uncertainty over cancer progression when pursuing non-definitive treatment. ${ }^{9}$ A recent multispecialty consensus conference spearheaded by the US Food and Drug Administration outlined a paradigm to evaluate PGA through a prospective randomized trial with men on active surveillance (AS) as a comparator. ${ }^{8}$ While the majority of urologists believe that PGA will become a standard option, ${ }^{10}$ patient attitudes and perceptions toward PGA have yet to be defined.

Currently, PGA is most commonly offered to men with low-risk to intermediate-risk PCa. ${ }^{11}$ Men with low-risk disease pursue AS due to evidence that grade group 1 cancers are often indolent in nature. ${ }^{9}$ Men with favorable intermediate-risk PCa are at higher risk of progression on surveillance but may also consider this management approach. ${ }^{12}$ While AS avoids the risk of side effects associated 
Key messages

What is already known about this subject?

- Partial gland ablation (PGA) is an emerging treatment option for prostate cancer (PCa) that allows for targeting of areas of biopsyproven $\mathrm{PCa}$ with the goal of minimizing treatment-related morbidity by avoiding treatment of non-cancerous areas of the prostate.

- We sought to explore patients' beliefs and attitudes toward PGA.

What are the new findings?

- We define treatment attributes that are significant to men with localized PCa, and one important theme is of the treatment intensity matching the severity of the disease.

- PGA appeals to men as middle ground that encompasses the duality of curative treatment and preservation of quality of life.

\section{How might these results affect future research or surgical practice?}

- The exploratory themes need further consideration and may be incorporated into shared decision-making discussions in men with low-risk disease, as PGA emerges as a treatment option.

with whole gland treatments, ${ }^{13}$ men on AS have reported the need to develop coping mechanisms related to anxiety over the long-standing chronicity of untreated disease. ${ }^{14}$ These accounts of patient experience on AS suggest there may be a role for PGA for some of these men.

In the shared decision-making process for PCa management, appropriate choice of treatment modality requires a comprehensive understanding of patient values and beliefs. To our knowledge, there has been a shift in the perspectives on $\mathrm{PCa}$ management by men with lower risk PCa away from definitive treatments and cancerspecific survival for preservation of urological functions and quality of life. ${ }^{15-18}$ Therefore, the goal of our study was to conduct qualitative interviews with men on AS to elucidate their consideration of treatment options and describe their opinions of PGA.

\section{SUBJECTS AND METHODS Approach}

The constant comparative method as described by Glaser and Strauss was used for thematic analysis. ${ }^{19}$ In this approach, data are first inductively coded and analyzed to identify concepts. As concepts evolve, data are revisited and undergo focused coding in an iterative process to develop themes. Interviews were conducted in a semistructured fashion to allow for the emergence of new patterns in data. Thematic saturation was initially observed after 15 interviews, when no new themes were identified. Interviews were conducted past the threshold of thematic saturation and concluded at 20 patients.

\section{Participants}

We identified English-speaking men on AS at Weill Cornell Medicine with very-low-risk, low-risk, and favorable intermediate-risk PCa consistent with the National Comprehensive Cancer Network guidelines. ${ }^{20}$ All men
Box 1 Summary on partial gland ablation (PGA)

\section{Summary}

- PGA is a novel approach to treating localized prostate cancer, meaning cancer that has not spread beyond a specific area. This treatment came about due to new imaging ability to visualize these specific areas in the prostate. PGA aims at destroying that area of the prostate only and leaving other areas untreated.

\section{Benefits}

- PGA is less invasive and comes with fewer side effects on urinary and sexual function than removing the whole prostate out of the body.

\section{Risks}

- PGA is not as curative or permanent of a treatment for prostate cancer as total removal is, and patients require further screening, testing, and treatment of any possible cancer in other areas of the prostate in the future. This includes additional biopsies and imaging, as received on active surveillance.

- Because PGA is so new, long-term outcomes are not known.

- Some payers do not cover PGA procedures because there is little evidence about it at the current moment.

had been seen in clinic at least once during the past 2 years.

\section{Recruitment and data collection}

All participants provided written informed consent. Eligible patients were mailed invitations to participate in the study and offered to be interviewed by mail or phone. Non-responders were contacted also by phone, when possible. All interviews were conducted by two research assistants uninvolved with medical care.

Prior to the interview segment on PGA, participants were provided a short summary of recent evidence-based findings (Box 1) as described by the EAU in 2018. ${ }^{2}$ As most patients had never heard of PGA before, this provided necessary context for discussion. Information was presented as objectively as possible. The mean duration of interviews was 32.1 (SD 8.0) min. Relevant medical information, including age, years since diagnosis, PSA, and Gleason score, was extracted from patient medical records after interviews.

The first 10 interviews were conducted with one researcher leading the interview and both researchers present at all times. These interviews served as a training period to promote standardization of process. The final 10 interviews were then conducted separately. All interviews were digitally recorded. The final iteration of the interview template is shown in Box 2.

\section{Analysis}

Interviews were transcribed, inductively coded and analyzed. Every five interviews, transcripts were reviewed with senior researchers to discuss concepts, perform focused coding, and develop themes. The interview template was then edited to reflect these emerging themes. Research assistants inductively coded and analyzed the first 10 transcripts together to standardize 


\section{Box 2 Interview template}

Could you tell me about when you received your diagnosis?

What were your main concerns at the time?

- Did your doctor recommend a specific treatment?

- Was there anything you didn't understand about what your doctor explained?

Who did you discuss this information with?

-What was important to you about your treatment options?

- Did you have any other medical conditions at the time that influenced your thinking?

- Have you heard of the term 'active surveillance' before?

- How do you feel right now living with low-grade prostate cancer on active surveillance?

- Have the factors you first considered at diagnosis changed or stayed the same after having been on active surveillance?

- Would anything lead to ending your active surveillance and changing treatment?

- Have you heard of partial gland ablation for prostate cancer?

- What is your impression of everything l've told you about partial gland ablation?

- If partial gland ablation were an option for you, what factors would influence your decision for or against pursuing it?

Would you consider pursuing partial gland ablation?

methodology and negotiate emerging themes. The final 10 transcripts were inductively coded and analyzed individually. Microsoft Excel was used for data management.

\section{RESULTS}

Out of 92 total men contacted, 20 consented for participation and were interviewed. Patient demographics and clinical characteristics are shown in table 1 . Of the subjects, $85 \%$ were white, $95 \%$ completed college or attained graduate degrees, and $70 \%$ had annual household income exceeding $\$ 110000$. Six (30\%) participants were diagnosed with very-low-risk, $12(60 \%)$ with low-risk, and $2(10 \%)$ with favorable intermediate-risk PCa. Four themes pertaining to men's pursuit of treatment options were identified: (1) the perception of psychological safety in the diagnosis of low-risk PCa; (2) the prioritization of minimally invasive options that have fewer side effects; (3) the dependence on the provider in the decisionmaking process; and (4) matching the aggressiveness of treatment with the degree of disease severity (table 2 ). Of the 20 men interviewed, 11 (55\%) expressed interest in pursuing PGA only if their cancer were to progress, while $9(45 \%)$ expressed interest at the current moment.

\section{Psychological safety in low-risk and favorable intermediate- risk $\mathrm{PCa}$}

Most men perceived their cancer as low risk, allowing them to find comfort in pursuing AS over definitive treatment. Although some men were first concerned at diagnosis, most came to understand their cancer as a chronic condition that they "needed to follow-up on," but not immediately treat because there was not a "huge chance of it spreading." One man acknowledged an "element
Table 1 Participant demographics and clinical characteristics

\begin{tabular}{lc}
\hline Median (IQR) age, years & $66(62.3-71.5)$ \\
\hline Median (IQR) years since diagnosis & $1.9(0.9-3.7)$ \\
\hline Median (IQR) PSA, ng/mL & $5.9(4.2-7.5)$ \\
Gleason score, $\mathrm{n}(\%)$ & \\
\hline $3+3$ & $18(90)$ \\
$3+4$ & $2(10)$ \\
\hline Race/ethnicity, $\mathrm{n}(\%)$ & \\
\hline White & $17(85)$ \\
\hline Black & $2(10)$ \\
\hline Asian & $1(5)$ \\
Educational attainment, $\mathrm{n}(\%)$ & \\
\hline High school degree & $1(5)$ \\
\hline Bachelor's degree & $10(50)$ \\
\hline Master's degree & $2(10)$ \\
\hline Doctoral degree & $7(35)$ \\
Household income, $\mathrm{n}(\%)$ & \\
\hline $60000-110$ 000 & $5(25)$ \\
\hline$>110000$ & $14(70)$ \\
\hline N/A & $1(5)$ \\
\hline Employment, $\mathrm{n}(\%)$ & $15(75)$ \\
\hline Full-time & $1(5)$ \\
\hline Part-time & $3(15)$ \\
\hline Retired & $1(5)$ \\
\hline Unemployed & $1(5)$ \\
\hline Marital status, $\mathrm{n}(\%)$ & \\
\hline Single & \\
\hline Married & \\
\hline Divorced & \\
\hline
\end{tabular}

N/A, not available; PSA, prostate-specific antigen.

of uncertainty" being on AS but did not feel the need to "dwell on" it. Moreover, one man claimed that his PCa "was the easiest thing I've had to deal with" among all his other medical conditions. Many men also perceived their cancer as non-fatal, commonly quoting that they were "more likely to die with it than of it." The ubiquity of cancer was often identified by multiple patients, with one man citing that he heard "if you're 70 you have a $70 \%$ chance of having PCa. Eighty is $80 \%$." One quote from an individual in his 60s exemplifies the lack of urgency most patients felt about their diagnosis:

I really don't think about it very much. To me, it's sort of on par with my hypertension... Hypertension, pre-diabetes, the prostate-and to me they're on a similar level... I think of them all on kind of the same... low level of health concern that I need to be careful about and watch and do the best I can. 
Discussions regarding PGA for patients accepting of AS yielded mixed results. While some embodied the idea of "just the fact that it exists does not make me want to go and get it," others expressed interest in further discussions at their next appointment.

Although most patients were satisfied with their current management, two particular men demonstrated a large psychological toll associated with their cancer. One patient in his 50s whose parent died from cancer described PCa as "this thing hanging over your head" and likened AS to "check[ing] in with the parole officer." Another interviewee described AS as "playing with fire" and "cutting corners." His thought process illustrates his feeling of brokenness associated with having PCa:

I really don't care how painful the procedure is. If they want to take a piece out, then take the piece out. If they blast me with radiation, that's what they gotta do... I don't feel like dying from cancer. That's been my only concern... I want it fixed... I don't want me walking around with cancer between my legs.

In these patients who were considering discontinuation of AS, PGA seemed "interesting" and something they wanted to discuss more with their physician.

\section{Preference for minimalism}

Most patients expressed preference for minimally invasive therapy when presented treatment options for their lowrisk PCa. Some men had an innate inclination toward a less invasive procedure without any definite reason. One patient in his mid-50s explained: "there is no scientific explanation... or no deep explanation" for wanting "to save a part of my body that can continue its function in my body."

For most men, the inclination toward the less invasive treatment reflected their desire to avoid side effects associated with prostatectomy. Most patients understood radical prostatectomy to be a "potentially life-changing procedure," naming loss of sexual and urinary functions as main reasons. Two of the youngest patients, in their mid-50s, detailed the desire to avoid "impotence" or "wearing a diaper." For one interviewee, the potential side effects were more worrisome than the uncertainty of living with cancer.

I was more worried about the consequences of surgery-the possible consequences- than... the actual diagnosis of cancer... the possibility of incontinence or... reduced or lower sexual activity.

Another patient echoed this sentiment toward PGA, stating "I would be willing to live with that uncertainty in order to avoid the side effects and invasiveness of the radical surgery." Many patients equated minimally invasive treatment with less side effects. For example, one man stated if treatment, including PGA, "is less invasive, it wouldn't have many side effects" and "will involve less risk for complication." Most men thought PGA was a securer 
alternative to radical prostatectomy and voiced plans to discuss with their urologist.

However, not all men found the minimally invasive option of PGA as intuitive. These men prioritized the treatment of cancer as more important than preservation of their urinary and sexual function. One individual in his 50s reflected on his confusion at the time of diagnosis with his cancer management: "the only thing that can happen is it can get worse, why don't we just do the procedure and get rid of it?" One patient, in his 60s, compared choosing between indefinitive treatment and possible urinary incontinence as having to pick his "poison," but found that "it's still better than ending up dead." Three men perceived PGA as a "band-aid" or a "temporary fix" as opposed to radical prostatectomy because of the remaining uncertainty after focal treatment.

Additionally, approximately a quarter of men interviewed touched on the invasiveness and frequency of periodic prostate biopsies while on AS. An interviewee in his 70s worried that prostate biopsies came with their own risk of sepsis and possible antibiotic resistance. Another patient in his 70s reported opting out of annual prostate biopsies in favor of non-invasive interventions with a naturopath. However, most men did not recognize prostate biopsies as a new disadvantage when transitioning to PGA from AS. One interviewee, in his late 50s, explained:

The negative of it is something that I'll have to do with active surveillance anyway. I still have to be monitored, I still have to do my biopsy, now I still have to do all of that. So... the negative part of it hasn't changed what I'm current doing.

While one patient expressed continued concern over prostate biopsies in regard to PGA, most men expressed sustained interest in learning more about PGA.

\section{The central role of the physician}

Many patients trusted and followed their urologists' recommendations in the decision-making process. Most men found confidence in physicians who were "up-todate" and "major," or renowned, in their specialty. Some men placed a high value in their physician's proficiency in the procedures they perform, stating "I have looked for the best surgeon to have the best outcome... regardless of the method or procedure I choose." Another individual highlighted his physician-patient relationship as reason for his trust: "I feel very comfortable with him and as long as I'm following the procedures that he told me to, I'm fine." Moreover, three men interviewed completely absolved themselves of personal research to rely on their physician's recommendations:

If my doctor says that's the best option, then I'll follow my doctor... If the doctor says yes, I'm not going to question him.

Most patients actively pursued information beyond the urology visit, usually online. Some men recounted anecdotes from peers with history of PCa, while a few turned to scientific studies. Many patients consulted with their friends and family, although most said this had no actual influence on their decision. Some individuals referred to their spouses as a "sounding board, someone [they] can bounce ideas off of" or "more of a supporting role [to] calm [them] down."

Most men asserted complete understanding of their disease and treatment options, as elucidated by their urologist. Patients believed their physicians were acting in their best interest, especially with the recommendation of a "non-invasive" and "less aggressive" approach. In addition, men found their urologist's recommendation of AS reinforced their appreciation of the low-risk nature of their condition and lessened the psychological distress associated with the cancer diagnosis. Furthermore, some men were encouraged by their primary urologists to pursue second opinions at a "teaching hospital and seek out a younger doctor." Few patients reported their optimistic genomic biopsy results reassuring them into AS. Men described a collaborative relationship with their urologists but felt themselves were ultimately in control of the decision:

No, my doctor and I made the decision. My family played, you know, a secondary. I make the decisions about my life; they don't. If the doctor says it is time for us to... need a decision, at that time, I will decide what needs to be done.

In considering PGA, men were even more inclined to depend on their urologists' recommendations and expertise, given the "experimental" nature of PGA.

My surgeon's confidence in it and his history of performing each of these procedures. I would only feel confident if he has a long history and has done it 800 times or so.

However, not all patients found their specialists to be candid in their treatment approach, stating "nobody really recommended any other treatment options other than the ones that they liked... or they performed." These patients felt the need to "inform myself of the different therapies that were open to me."

\section{Intensity of treatment parallels disease severity}

Many patients described their decision to pursue AS as a logical process whereby low-risk disease was appropriately managed by observation. AS was described as "an apt measure based on the diagnosis" compared with the "over-reactive invasive" option of radical prostatectomy. As one patient explained, "I just felt like it was the right thing to do. It made sense to me. It was logical... as long as there's... regular and consistent monitoring... you know, it seemed that's what I should do." The lack of symptoms of low-risk disease also played a role in deciding against definitive treatment in a patient whose follow-up biopsy was negative: "If my prostate is working... If I' $m$ not having any of the symptoms or I'm not having any discomfort... Why would I go and do anything radical?" One patient 
who also saw a naturopath for his PCa even felt that the management process of AS itself was excessive:

It's our culture that's leading us to-let's do this procedure, let's do this test and that test and more... what is the bottom-line improvement in one's life when we're, you know, talking about something that's not that aggressive.

As a result, many patients expressed that they would proceed to surgery or radiation only if it were "necessary" or if the cancer were "worsening," which they felt were determined by the combination of test results and provider recommendation.

This stepwise approach was expressed similarly in discussions regarding PGA, although with varied outcomes. Eleven men (55\%) expressed interest in pursuing PGA only if their cancer were to progress, while nine men (45\%) expressed interest at the current moment. In considering further discussion on PGA with their provider, some expressed interest only if future biopsies showed worse outcomes, since "the treatment would be aggressive to match the more aggressive diagnosis." A few examples are illustrated:

I'm actually happy with the active surveillance. Someone would have to say that 'your risk has increased' and if there were something that said 'your risk has increased' then I would prefer to have something like focal therapy or focal surgery than a full excision of the prostate. I think that I would need a really bad Gleason score to opt in for some kind of treatment. It's gotta be a real black and white issue.

Yet others felt that the relatively conservative and localized approach of PGA deserved consideration and at least a discussion with their provider, even at their current risk category.

The finding was in a very small area. And that the Gleason score was not highly aggressive. So therefore... it would make sense to me to do the focal therapy as opposed to doing something more radical.

Given the ability to focus on the areas of concern, just those parts of the prostate... might suggest cancer could be targeted fairly well. You know, I'd be game for something like that.

\section{DISCUSSION}

Our study identifies four key thought processes in considering PGA among men with very-low-risk, low-risk, and favorable intermediate-risk PCa on AS, namely the perception of psychological safety in a lower risk diagnosis, the prioritization of minimally invasive options with fewer side effects, the dependence on the provider in the decision-making process, and the consideration of treatment aggressiveness with respect to disease severity. These concepts were further explored in the context of considering PGA as a treatment option, for incorporation into shared decision-making discussions. Our cohort reflects recent treatment expansion of AS to include men with favorable intermediate-risk PCa along with men with verylow-risk and low-risk PCa. ${ }^{8}$ Overall, 9 patients expressed interest in speaking with their provider to discuss PGA at their current risk category and 11 expressed interest in the event their cancer progresses.

Most men described a feeling of psychological safety with having low-risk disease on AS. This finding parallels other studies that identified men's acute perception of the low-risk category and non-immediate threat to life. ${ }^{1321}$ However, some men in our study expressed a significant discomfort with the uncertainty of their diagnosis and were considering other treatment options. This uncertainty has been observed by others and shown to be associated with lower quality of life. ${ }^{22}$ Men expressing uncertainty about AS were more receptive to PGA, and many patients comfortable on AS were also interested in further discussion at their current risk level.

Earlier studies of AS assert that patients with low-risk PCa valued prolonged survival over preservation of urinary and sexual functions, ${ }^{1718}$ thus are likely to pursue definitive treatment over AS. ${ }^{23}{ }^{24}$ However, our findings concur with more recent findings that indicate a cultivating preference for minimally invasive treatments by men with a refined understanding of their low-risk condition. ${ }^{1315}$ Currently it is widely known that men with lowrisk PCa managed on AS have higher quality of life, ${ }^{25}$ explaining men's motivation to trade off low uncertainty over survival for preservation of urinary function. ${ }^{15}$ PGA was appealing as a curative alternative to AS and a less invasive option to radical prostatectomy for men in this study. However, $15 \%$ of men interviewed perceived PGA as a temporary cure when compared with whole gland treatments as the uncertainty of recurrence remains.

Trust in the physicians and their recommendations has been shown to play a key role in pursuing any treatment strategy, ${ }^{9} 2627$ although some men also value playing an active role ${ }^{28}$ and taking ownership over their choices. ${ }^{132729}$ Regardless, greater patient knowledge is also associated with greater decision-making difficulty, ${ }^{30}$ which suggests an important role in shared decision-making with providers. Our study is consistent with prior findings that patients who established a collaborative relationship with their physicians ${ }^{31}$ are more likely to seek their physician's expertise and 'decisional support' ${ }^{29}$ when surveying new treatment options, including PGA. However, few patients did not find the recommendations for focal treatments to be candid, as they found specialists predisposed to recommending procedures they primarily perform.

Many men identified a logical process in their decisionmaking in which their low-risk disease was appropriately managed by the observational nature of AS. Previous studies have identified men's understanding of the rationale behind AS as a contributing factor toward staying on AS. ${ }^{21}$ Our findings contribute that men on AS respect the escalation of treatment invasiveness that parallels disease progression. This novel concept of treatment intensity 
matching disease can be explored in shared decisionmaking discussions with the introduction of PGA as an intermediate option in the PCa treatment spectrum. Interestingly, these men differed in their opinions on where PGA stands in this hierarchy, with some finding it an appropriate option for low-risk disease and others believing it is only necessary for higher risk disease.

Earlier studies found men with low-risk PCa having higher overall satisfaction with care with definitive treatments than with AS. ${ }^{31}$ However, definitive treatments for patients with low-risk localized PCa are currently considered overtreatment and introduce unnecessary side effects. ${ }^{23}$ Nevertheless, many men elect to undergo definitive therapy to address the uncertainty of cancer. ${ }^{17}$ Although short-term oncological outcomes vary and may depend on the specific modality used, ${ }^{2} 32$ PGA emerges as middle ground that encompasses the satisfaction of curative treatment and preservation of quality of life. ${ }^{23}$ Additional research on men's preferences and attitudes focused on each treatment modality under PGA, including high-intensity focused ultrasound, irreversible electroporation, cryotherapy, photodynamic therapy, and focal laser thermal ablation, will further guide shared decision-making for PGA.

This study is not without limitations. First, this was a qualitative study of 20 men on AS from a single tertiary care center located in New York City. Patients in this study were mostly white educated men residing in areas of higher income. Thus, our findings may not be generalizable to men from different backgrounds. Additionally, sampling bias must be considered given that our cohort includes only men who volunteered to be interviewed. Second, our study concerns men with lower risk PCa who never received treatment on AS. However, inclusion of men of similar risk category with recent diagnosis undecided on a treatment plan and men with history of PCa treatment still eligible for PGA would provide a more comprehensive insight into the perceptions and attitude of all eligible men for PGA, not just those on AS. Moreover, the time since diagnosis averaged 2.6 years in our study and may have contributed to recall bias, loss of details about the initial consultation, and establishment of comfort regarding their diagnosis and treatment options. Additionally, semistructured interviews required probing patients with unprompted questions, adding to both interviewer and response bias. Lastly, phone interviews do not provide visual cues and may contribute to the loss of non-verbal data and contextual information and misinterpretation of responses. ${ }^{33}$ However, phone interviews offer facial anonymity, which may empower patients to disclose sensitive information more readily. ${ }^{33}$ Of note, 2 out of 20 men interviewed (10\%) had a Gleason score of $3+4$. While these patients serve to reflect the recent change in treatment paradigm to include men of favorable intermediate-risk PCa in AS, they are not representative of the entire group of men with PCa on AS.

In conclusion, as PGA develops there has been an emerging consensus that men with tumor characteristics eligible for AS may be the best candidates for PGA; however, there is little knowledge of men's attitudes and perspectives on PGA. Herein we identify four themes and their relation to men's considerations of PGA: the feeling of psychological safety associated with low-risk disease, a preference for minimally invasive treatment, the central role of the physician, and the pursuit of treatment option intensity that parallels disease severity. In a small sample size of highly educated men with low-risk PCa that is experienced with AS, we demonstrate that almost half of men have potential interest in PGA, despite low-grade evidence concerning intermediate and long-term outcomes.

Twitter Eliza Cricco-Lizza @DrCriccoLizza, Spyridon Basourakos @SBasourakos and Jim Hu @jimhumd

Contributors All authors have contributed significantly to the study design, collection of data, analysis, and/or manuscript preparation.

Funding Jim C. Hu receives research support from the Frederick J. and Theresa Dow Wallace Fund of the New York Community Trust. Jim C. Hu also receives salary support from NIH R01 CA241758, PCORI CER-2019C1-15682 and CER2019C2-17372. The remaining authors report no further disclosures related to this work.

Competing interests None declared.

Patient consent for publication Not required.

Ethics approval Ethical approval was obtained from the Weill Cornell Institutional Review Board.

Provenance and peer review Not commissioned; externally peer reviewed.

Data availability statement All data relevant to the study are included in the article.

Open access This is an open access article distributed in accordance with the Creative Commons Attribution Non Commercial (CC BY-NC 4.0) license, which permits others to distribute, remix, adapt, build upon this work non-commercially, and license their derivative works on different terms, provided the original work is properly cited, appropriate credit is given, any changes made indicated, and the use is non-commercial. See: http://creativecommons.org/licenses/by-nc/4.0/.

\section{ORCID iDs}

Sonia Hur http://orcid.org/0000-0003-2231-0918

Jim Hu http://orcid.org/0000-0003-2562-8024

\section{REFERENCES}

1 Demirel C, Altok M, Davis J. Focal therapy for localized prostate cancer: is there a "middle ground" between active surveillance and definitive treatment? Asian J Androl 2019;21:37.

2 van der Poel HG, van den Bergh RCN, Briers E, et al. Focal therapy in primary localised prostate cancer: the European association of urology position in 2018. Eur Urol 2018;74:84-91.

3 Ahdoot M, Lebastchi AH, Turkbey B, et al. Contemporary treatments in prostate cancer focal therapy. Curr Opin Oncol 2019;31:200-6.

4 Perera M, Krishnananthan N, Lindner U, et al. An update on focal therapy for prostate cancer. Nat Rev Urol 2016;13:641-53.

5 Lodeizen O, de Bruin M, Eggener S, et al. Ablation energies for focal treatment of prostate cancer. World J Urol 2019;37:409-18.

6 Azzouzi A-R, Vincendeau S, Barret E, et al. Padeliporfin vasculartargeted photodynamic therapy versus active surveillance in men with low-risk prostate cancer (CLIN1001 PCM301): an open-label, phase 3, randomised controlled trial. Lancet Oncol 2017;18:181-91.

7 Basourakos SP, Al Hussein Al Awamlh B, Bianco FJ, et al. Feasibility of in-office MRI-targeted partial gland cryoablation for prostate cancer: an ideal stage 2A study. BMJ Surg Interv Health Technologies 2020;2:e000056.

8 Weinstock C, Suzman D, Kluetz P, et al. Development of treatments for localized prostate cancer in patients eligible for active surveillance: U.S. food and drug administration oncology center of excellence public workshop. J Urol 2020;203:115-9.

9 Mallapareddi A, Ruterbusch J, Reamer E, et al. Active surveillance for low-risk localized prostate cancer: what do men and their partners think? Fam Pract 2017;34:90-7. 
10 Marra G, Ploussard G, Ost P, et al. Focal therapy in localised prostate cancer: real-world urological perspective explored in a crosssectional European survey. Urol Oncol 2018;36:529.e11-529.e22.

11 Klotz L, Emberton M. Management of low risk prostate cancer-active surveillance and focal therapy. Nat Rev Clin Oncol 2014;11:324-34.

12 Cooperberg MR, Carroll PR. Trends in management for patients with localized prostate cancer, 1990-2013. JAMA 2015;314:80.

13 Davison BJ, Oliffe JL, Pickles T, et al. Factors influencing men undertaking active surveillance for the management of low-risk prostate cancer. Oncol Nurs Forum 2009;36:89-96.

14 Mader EM, Li HH, Lyons KD, et al. Qualitative insights into how men with low-risk prostate cancer choosing active surveillance negotiate stress and uncertainty. BMC Urol 2017;17:35.

15 Watson V, McCartan N, Krucien N, et al. Evaluating the tradeoffs men with localized prostate cancer make between the risks and benefits of treatments: the compare study. $J$ Urol 2020;204:273-80.

16 de Bekker-Grob EW, Bliemer MCJ, Donkers B, et al. Patients' and urologists' preferences for prostate cancer treatment: a discrete choice experiment. Br J Cancer 2013;109:633-40.

17 Mazur DJ, Hickam DH. Patient preferences for management of localized prostate cancer. West J Med 1996;165:26-30.

18 Knight SJ, Latini DM. Sexual side effects and prostate cancer treatment decisions: patient information needs and preferences. Cancer J 2009;15:41-4.

19 Glaser B, Strauss A. The Discovery of Grounded Theory: Strategies for Qualitative Research. Chicago: Aldine Transaction, 1967. ISBN: 0-202-30260-1.

20 National Comprehensive Cancer Network. NCCN clinical practice guidelines in oncology: prostate cancer. version 2, 2020. Available: https://www.nccn.org/professionals/physician_gls/pdf/prostate.pdf [Accessed 25 May 2020].

21 Seaman AT, Taylor KL, Davis K, et al. Why men with a low-risk prostate cancer select and stay on active surveillance: a qualitative study. PLoS One 2019;14:e225134.
22 Hegarty JM, Wallace M, Comber H. Uncertainty and quality of life among men undergoing active surveillance for prostate cancer in the United States and ireland. Am J Mens Health 2008;2:133-42.

23 Barqawi AB, Krughoff KJ, Eid K. Current challenges in prostate cancer management and the rationale behind targeted focal therapy. Adv Urol 2012;2012:1-7.

24 Zeliadt SB, Ramsey SD, Penson DF, et al. Why do men choose one treatment over another? Cancer 2006;106:1865-74

25 Lardas M, Liew M, van den Bergh RC, et al. Quality of life outcomes after primary treatment for clinically localised prostate cancer: a systematic review. Eur Urol 2017;72:869-85.

26 Scherr KA, Fagerlin A, Hofer T, et al. Physician recommendations Trump patient preferences in prostate cancer treatment decisions. Med Decis Making 2017;37:56-69.

27 Davison BJ, Breckon E. Factors influencing treatment decision making and information preferences of prostate cancer patients on active surveillance. Patient Educ Couns 2012;87:369-74.

28 Saigal CS, Lambrechts SI, Seenu Srinivasan V, Srinivasan S V, et al. The voice of the patient methodology: a novel mixed-methods approach to identifying treatment goals for men with prostate cancer Patient 2017;10:345-52.

29 Orom H, Biddle C, Underwood W, et al. What Is a "Good" Treatment Decision? Decisional Control, Knowledge, Treatment Decision Making, and Quality of Life in Men with Clinically Localized Prostate Cancer. Med Decis Making 2016;36:714-25.

30 Daum LM, Reamer EN, Ruterbusch JJ, et al. Patient knowledge and qualities of treatment decisions for localized prostate cancer. J Am Board Fam Med 2017;30:288-97.

31 Denberg TD, Melhado TV, Steiner JF. Patient treatment preferences in localized prostate carcinoma: the influence of emotion, misconception, and anecdote. Cancer 2006;107:620-30.

32 Marshall S, Taneja S. Focal therapy for prostate cancer: the current status. Prostate Int 2015;3:35-41.

33 Novick G. Is there a bias against telephone interviews in qualitative research? Res Nurs Health 2008;31:391-8. 